\title{
Serotonin Discharge Regulation by Additional Neurotransmitters of Rat Hippocampus Associated With the Continence Central Circuit
}

\author{
Jae Heon Kim¹, Young Soo Ahn², Yun Seob Song ${ }^{1}$ \\ ${ }^{1}$ Department of Urology, Soonchunhyang University College of Medicine, Seoul, Korea \\ ${ }^{2}$ Department of Pharmacology, Yonsei University College of Medicine, Seoul, Korea
}

Purpose: The lower urinary tract is believed to be centrally regulated with the involvement of a range of neurotransmitters. The parasympathetic excitatory input to the urinary bladder is suppressed when the serotonergic system is activated, and thereby voiding is blocked. In healthy people, continence is usually underpinned by hippocampal formation (circuit 3). In order to advance knowledge of how serotoninergic neurons and additional nerve fibers were correlated, the purpose of the present work was to research how the discharge of serotonin from hippocampal slices was affected by different neurotransmitters in rat models.

Methods: The adopted procedure involved administration of the central neurotransmitters acetylcholine, norepinephrine, dopamine, N-methyl-D-aspartate (NMDA), gamma-aminobutyric acid (GABA), glycine, and neuropeptide $\mathrm{Y}$ as well as monitoring of the alterations in the discharge of $\left[{ }^{3} \mathrm{H}\right] 5$-hydroxytryptamine (5-HT). Furthermore, to determine whether the effect of the neurotransmitters was influenced by interneuron, tetrodotoxin was also employed.

Results: Acetylcholine $\left(10^{-5} \mathrm{M}\right)$ did not alter $\left[{ }^{3} \mathrm{H}\right] 5-\mathrm{HT}$ discharge, whereas more 5-HT was discharged from the hippocampal slices of rats under stimulation by norepinephrine $\left(10^{-5} \mathrm{M}\right)$ as well as dopamine $\left(10^{-5} \mathrm{M}\right)$ and tetrodotoxin $\left(10^{-6} \mathrm{M}\right)$ did not inhibit the discharge. By contrast, tetrodotoxin inhibited the discharge of $\left[{ }^{3} \mathrm{H}\right] 5-\mathrm{HT}$ that was exacerbated by NMDA $\left(10^{-4} \mathrm{M}\right)$. Meanwhile, compared to control, GABA $\left(10^{-5} \mathrm{M}\right)$, glycine $\left(10^{-5} \mathrm{M}\right)$, or neuropeptide $\mathrm{Y}\left(10^{-6} \mathrm{M}\right)$ did not alter the $\left[{ }^{3} \mathrm{H}\right] 5-\mathrm{HT}$ discharge.

Conclusions: From the research findings, it can be concluded that 5-HT discharge from rat hippocampus is enhanced by norepinephrine and dopamine through direct effect on the 5-HT neuronal terminal. By contrast, 5-HT discharge is intensified by NMDA by activating interneurons.

Keywords: Serotonin; Hippocampal slices; N-methyl-D-aspartate; Norepinephrine; Dopamine

- Fund/Grant Support: This research was supported by grant from Soonchunhyang University Research Fund and the National Research Foundation of Korea (NRF) funded by the Ministry of Education, Science and Technology (2021R1A2C1004163).

- Research Ethics: This research was approved by the Institutional Animal Care and Use Committee of Soonchunhyang University, Seoul Hospital (IRB No. 2019-4). The authors carried out the experimental protocols, in line with the National Institute of Health Guide for the Care and Use of Laboratory Animals.

- Conflict of Interest: No potential conflict of interest relevant to this article was reported.

\footnotetext{
- HIGHLIGHTS

- A range of different neurons was involved in 5-HT discharge from rat hippocampal slices.

- 5-HT discharge was intensified by norepinephrine and dopamine through direct action on the 5-HT neuronal terminal.

- 5-HT discharge was intensified by NMDA in an indirect manner, via interneuron mediation.
}

Corresponding author: Yun Seob Song (iD https://orcid.org/0000-0002-0909-3341 Department of Urology, Soonchunhyang University Seoul Hospital, Soonchunhyang University School of Medicine, 59 Daesagwan-ro, Yongsan-gu, Seoul 04401, Korea

Email: yssong@schmc.ac.kr

Submitted: November 2, 2021 / Accepted after revision: November 18, 2021
This is an Open Access article distributed under the terms of the Creative Commons Attribution Non-Commercial License (http://creativecommons.org/licenses/by-nc/4.0/) which permits unrestricted non-commercial use, distribution, and reproduction in any medium, provided the original work is properly cited. 


\section{INTRODUCTION}

The lower urinary tract is thought to be centrally regulated by different neurotransmitters. Evidence suggests that the spinal and supraspinal reflex pathways controlling the bladder and external urethral sphincter are dependent on the neurotransmitter glutamate, which exerts its effect on both N-methyl-D-aspartate (NMDA) and non-NMDA receptors. Meanwhile, the activated receptor type, the site where the receptors are found in the brain, and the species are the determinants of the effect (inhibition or excitation) of certain neurotransmitters, including dopamine and acetylcholine (Ach). Tonic suppressive regulation is presented by inhibitory amino acids gamma-aminobutyric acid (GABA) and glycine within the pontine micturition center (PMC), modulating the capacity of the bladder [1-3].

A major neurotransmitter that blocks central control is serotonin (5-hydroxytryptamine, 5-HT). Serotonergic innervation of high density characterizes the spinal reflex circuits with a role in the voiding activity. Voiding can be inhibited when the central serotonergic system is activated as this blocks the parasympathetic excitatory input to the urinary bladder. Furthermore, thoracic sympathetic preganglionic neurons are activated long term by 5 -HT. Research on cats has shown that reflex bladder activity is suppressed when the raphe nuclei are stimulated. Furthermore, the sacral parasympathetic nucleus contains 5-HT1A and 5-HT2 receptors. Cat micturition is suppressed when 5HT1A receptors are activated whereas rat micturition is unaffected [4-6]. Serotonergic neurons are present in medullary raphe nuclei, which are among the brain neurons that participate in the regulation of the bladder, urethra, and urethral sphincter [7]. Nevertheless, knowledge is limited regarding the network of different neurotransmitters involved in the central regulation of serotonin.

Sacral preganglionic neurons or the area of the sacral dorsal commissure are supplied directly with synaptic inputs by neurons in PMC. These neurons are considered to play a significant role in facilitating the effect of excitation or suppression on voiding or continence [8]. In healthy people, the gradual filling of the bladder is usually accompanied by the activation of the subcortical network, including the periaquidcutal gray and parahippocampal cortex, with no sensation (circuit 3). It seems that this process occurs without the participation of the cortical areas. The circuit is especially related to the safety emotional facets of voiding given the nearness of the parahippocampal cortex to the amygdala. More specifically, the circuit may use the presumed safe signal of continence from the hypothalamus to deliver output to the brainstem nuclei [5,9]. Extensive research has been conducted on the internal and external links of nerve cells in the hippocampus.

Regarding the manner in which the nerves are distributed within the hippocampus, the locus coeruleus and the raphe nucleus are respectively the entry points for the noradrenalinergic nerve fibers and the serotonergic nerve fibers. The majority of the hippocampal regions, particularly the dentate gyrus, are traversed by these 2 types of nerve fibers. Furthermore, the hippocampus also includes dopaminergic nerve fibers from the ventral tegmental region, while the medial septal nucleus is the source of the cholinergic nerve fibers, GABAergic nerve fiber, and glutaminergic nerve fibers entering the hippocampus. Moreover, the dentate gyrus, hippocampal CA1, subiculum, and the polymorphic layers of the entorhinal cortex all contain the neuropeptide $\mathrm{Y}[10,11]$.

Fiber-derived neurotransmitters are capable of modulating the discharge of additional neurotransmitters. Serotonin discharge from serotonergic nerve fibers originating in raphe nuclei or corpus striatum was diminished by glutamate, while suppression of glutamate prevented such an effect. Furthermore, both tetrodotoxin and bicuculline, a suppressor of GABA, inhibited glutamate from reducing 5-HT. This led to the proposition that GABA interneuron exerted negative modulation of 5-HT discharge from the serotonergic nerve fiber terminal in rat hippocampus [12].

To comprehend the action of the micturition-regulating central circuits and the manner in which they can be modulated to manage bladder dysfunction, it is necessary to understand the neurochemical signals in these circuits. On this basis, it may be possible to devise new pharmacological treatment interventions.

In the context of the brain slices isolated empirically, there is clear understanding of how hippocampal neurons are synaptically connected and the in vivo qualities displayed by such connections, with strict control of temperature, oxygen pressure, and $\mathrm{pH}$. For these reasons, hippocampal slices are good materials for experiments [13]. The way in which the unplanned discharge of 5-HT from the hippocampal slices of rats was influenced by a number of neurotransmitters was documented in the present work. Moreover, to explore interneuron participation, the impact of tetrodotoxin on the effect of the neurotransmitters was analyzed as well. 


\section{MATERIALS AND METHODS}

\section{Hippocampal Slice Preparation}

All study protocols were performed in keeping with the $\mathrm{Na}$ tional Institute of Health Guide for the Care and Use of Laboratory Animals (2001); they were sanctioned by the facility's Institutional Animal Care and Use Committee.

The empirical work employed Sprague-Dawley rats of adult age and male sex and weighing 150-200 g. They were acclimatized to laboratory conditions for a minimum of one week. After decapitation, the extraction of the brain of every rat was conducted quickly. The brain was separated into its 2 halves at midline. A McIlwain mechanical tissue chopper (The Mickle Laboratory Engineering Co., Gomshall, Surrey, UK) was used for $400-\mu \mathrm{m}$ transverse slicing of the hippocampus from both brain halves. Each one of these processes was carried out on ice and ice-cold $\left(2^{\circ} \mathrm{C}-4^{\circ} \mathrm{C}\right)$ standard incubation medium was used for repetitive washing of the tissues. The composition of the standard incubation medium included $124 \mathrm{mM}$ of $\mathrm{NaCl}, 4 \mathrm{mM}$ of $\mathrm{KCl}, 2 \mathrm{mM}$ of $\mathrm{CaCl}_{2}, 1 \mathrm{mM}$ of $\mathrm{MgSO}_{4}, 1.25 \mathrm{mM}$ of $\mathrm{KH}_{2} \mathrm{PO}_{4}$, $25 \mathrm{mM}$ of $\mathrm{NaHCO}_{3}$, and $10 \mathrm{mM}$ of glucose, with a $\mathrm{pH}$ of 7.3-7.5 and $95 \% \mathrm{O}_{2} / 5 \% \mathrm{CO}_{2}$. The metabolic degeneration of 5-HT was avoided through the addition of $12.5 \mu \mathrm{m}$ of nialamide (Sigma Chemical Co., St. Louis, MO, USA).

\section{Empirical Procedure}

To reach equilibrium, 6 hippocampal slices from the rats were kept in standard incubation medium with a temperature of $37^{\circ} \mathrm{C}$ and $95 \% \mathrm{O}_{2} / 5 \% \mathrm{CO}_{2}$ saturation for half an hour. This was followed by substitution of the incubation medium with fresh medium comprising $\left[{ }^{3} \mathrm{H}\right] 5-\mathrm{HT}(0.1 \mu \mathrm{M}, 74 \mu \mathrm{Ci}$; Amersham International plc., Buckinghamshire, UK) for uptake. The slices were incubated for 20 minutes and then washed thrice with standard incubation medium. The next step was arbitrary separation of the slices and transfer to 6 glass vials with a volume of incubation medium of $3 \mathrm{~mL}$. Re-uptake all through the experiment was prevented through the addition of $10 \mu \mathrm{m}$ of zimelidine (Research Biochemical International, Natick, MA, USA) to the medium. The content of every vial was removed and an identical amount of new medium was added every 10 minutes for a period of 100 minutes. The removed content helped to measure how radioactive the discharged 5-HT was. The initial 50 minutes of the procedure were followed by the administration of the testing pharmaceutical agents at the sixth and seventh 10-minute intervals, respectively. Administration of tetro- dotoxin was done all through the experiment (0-100 minutes).

\section{Measurement of $\left[{ }^{3} \mathrm{H}\right] 5-\mathrm{HT}$ Radioactivity}

A mixture was produced from $1 \mathrm{~mL}$ of medium removed from vial at 10-minute intervals and $9 \mathrm{~mL}$ of liquid scintillation cocktail (READY SAFE, Beckman Instruments Inc., Fullerton, CA, USA). A liquid scintillation counter (Beckman Instruments Inc.) was employed to measure the mixture radioactivity. Furthermore, $1 \mathrm{~mL}$ of tissue solubilizer (SOLUENE, Pachard Instrument Co., Inc., Downers Grove, IL, USA) was applied to the tissues, followed by 2 -hour incubation at $37^{\circ} \mathrm{C}$ in order to determine how radioactive the $\left[{ }^{3} \mathrm{H}\right] 5$-HT remaining on the hippocampal slices was. The tissue solubilizer was subsequently neutralized through addiction of $70 \mu \mathrm{L}$ of glacial acetic acid (Merk \& Co., Inc., Kenilworth, NJ, USA). Moreover, for measurement purposes, a mixture was created from $100 \mu \mathrm{L}$ of the fully solubilized specimen and $9 \mathrm{~mL}$ of liquid scintillation cocktail.

\section{Pharmaceutical Agents}

Sigma Chemical Co. supplied the Ach, norepinephrine, dopamine, NMDA, GABA, glycine, neuropeptide Y, tetrodotoxin, and nialamide, while Amersham International plc. and Research Biochemicals International respectively supplied $\left[{ }^{3} \mathrm{H}\right] 5$ hydroxytryptamine and zimelidine $\mathrm{HCl}$. Packard Instrument Company Inc. (Downers Grove, IL, USA) supplied the tissue solubilizer Soluen 350 and Beckman Instruments Inc. (Downers Grove, IL, USA) supplied the liquid scintillation cocktail Ready Safe.

\section{Analysis of Data}

The expression of every result took the form of fractional release. The applied definition of fractional release (FR) was:

$$
\operatorname{FR}(\%)=\frac{c p m(\text { medium }) F R \times 100}{c p m(\text { medium }) T L+c p m(\text { tissue })}
$$

Cpm (medium) FR denoted the radioactivity (counts per min, cpm) of $\left[{ }^{3} \mathrm{H}\right] 5$-HT discharged into the media collected during the specified interval of 10 minutes; cpm (medium) TL denoted the totality of the radioactivity (cpm) of $\left[{ }^{3} \mathrm{H}\right] 5-\mathrm{HT}$ discharged into the media collected at 10-minute intervals from the established time fraction to the final point of the period of observation; and cpm (tissue) denoted the radioactivity (cpm) of $\left[{ }^{3} \mathrm{H}\right] 5$ HT lefton hippocampal slices following the period of observation of 100 minutes. 
Analysis of variances with subsequent Scheffe test was conducted to statistically compare the different groups, with the significance level being established at 0.05 .

\section{RESULTS}

\section{The Discharge of [ $\left.{ }^{3} \mathrm{H}\right] 5-\mathrm{HT}$ in Control Group and Group With Prior Administration of Tetrodotoxin in Conditions of Standard Media}

5-HT was discharged spontaneously from the slices of hippocampus all through the empirical work. The initial 40 minutes saw a fast reduction in the discharge of 5-HT, followed by stable discharge until the end of the observation period (100 minutes). Furthermore, the unplanned 5-HT discharge was unaffected by tetrodotoxin $\left(10^{-6} \mathrm{M}\right)$ administration, while the administration of the pharmaceutical agents was done for 20 minutes at the sixth and seventh 10-minute intervals. The chosen datum point was the discharge of 5-HT at the fifth 10-minute interval and the expression of alterations in the discharge took the form of percent values contrasted against the fifth 10minute interval (Fig. 1).

\section{Norepinephrine- or Dopamine-Induced Alteration in $\left[{ }^{3} \mathrm{H}\right] 5-\mathrm{HT}$ Discharge}

Norepinephrine and dopamine were employed to investigate how the unplanned discharge of 5-HT from the slices of hippo- campus was affected by the adrenergic action.

By comparison to control, norepinephrine $\left(10^{-6} \mathrm{M}\right)$ induced a markedly higher discharge of 5-HT at both the sixth 10-minute interval $(115.7 \% \pm 2.3 \%)$ and the seventh 10 -minute interval $(105.8 \% \pm 5.5 \%)(\mathrm{P}<0.05)$. Similarly, more 5-HT was discharged from the slices of hippocampus under the action of dopamine. In fact, as shown in Fig. 2, dopamine elicited a more intense discharge of 5-HT compared to norepinephrine. Additionally, the discharge of 5-HT remained at a heightened level for an extra 20 minutes following dopamine removal from the incubation medium.

The action of norepinephrine and dopamine was analyzed following tetrodotoxin $\left(10^{-6} \mathrm{M}\right)$ administration in order to determine if interneuron involvement was the reason why norepinephrine or dopamine enhanced 5-HT discharge. It was thus found that 5-HT discharge from hippocampal slices triggered by norepinephrine and dopamine was unaffected by tetrodotoxin (Figs. 2 and 3).

\section{NMDA-Induced Alteration in $\left[{ }^{3} \mathrm{H}\right] 5-\mathrm{HT}$ Discharge}

The action of NMDA was investigated to assess the glutamatergic impact on the unplanned discharge of 5-HT from the slices of hippocampus. As illustrated in Fig. 2, compared to control, NMDA $\left(10^{-4} \mathrm{M}\right)$ induced a markedly higher discharge of 5-HT after both 10 minutes of treatment $(122.5 \pm 2.4 \%)$ and after 20 minutes of treatment $(105.0 \pm 3.4 \%)(\mathrm{P}<0.05)$. However, the

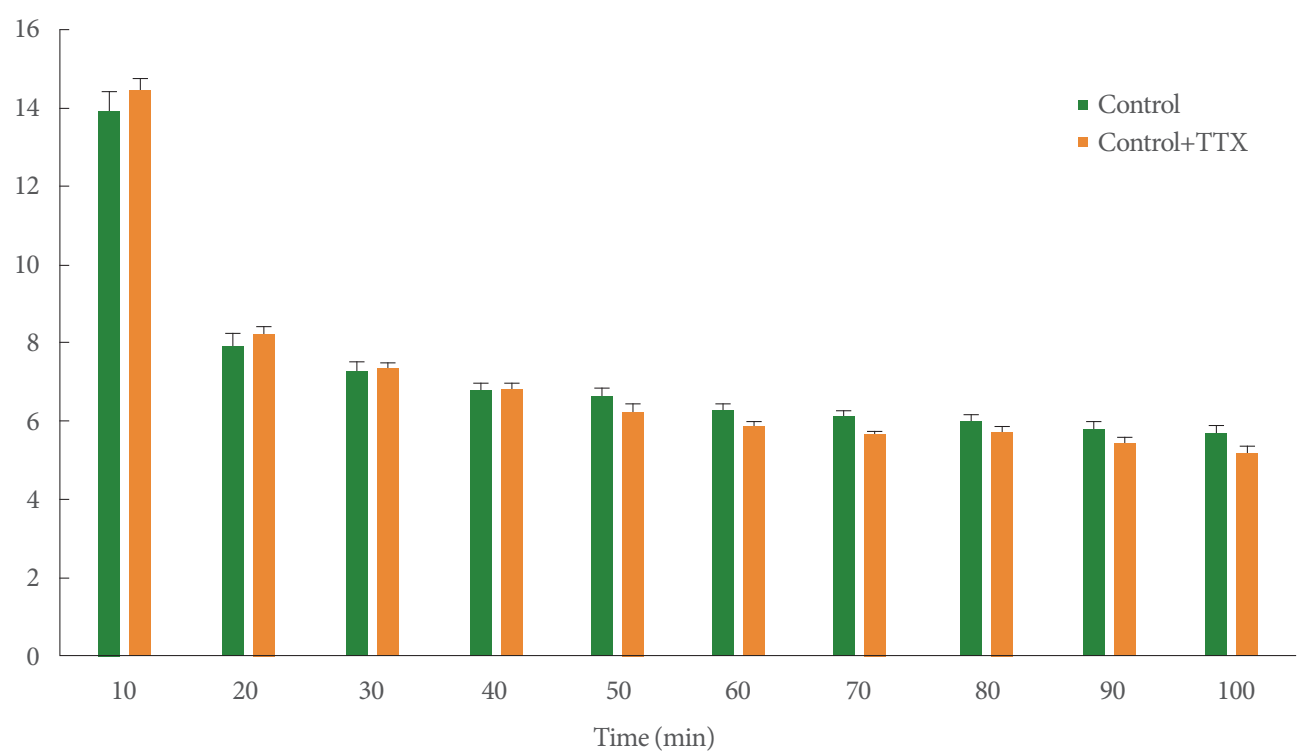

Fig. 1. The impact of tetrodotoxin on the unplanned discharge (cpm) of 5-hydroxytryptamine (5-HT) from the hippocampal slices of rats. Administration of tetrodotoxin continued all through the empirical work. In the initial 40 minutes, there was a fast reduction in 5-HT discharge, while subsequently 5-HT discharge became stable up to 100 minutes. TTX, tetrodotoxin. 


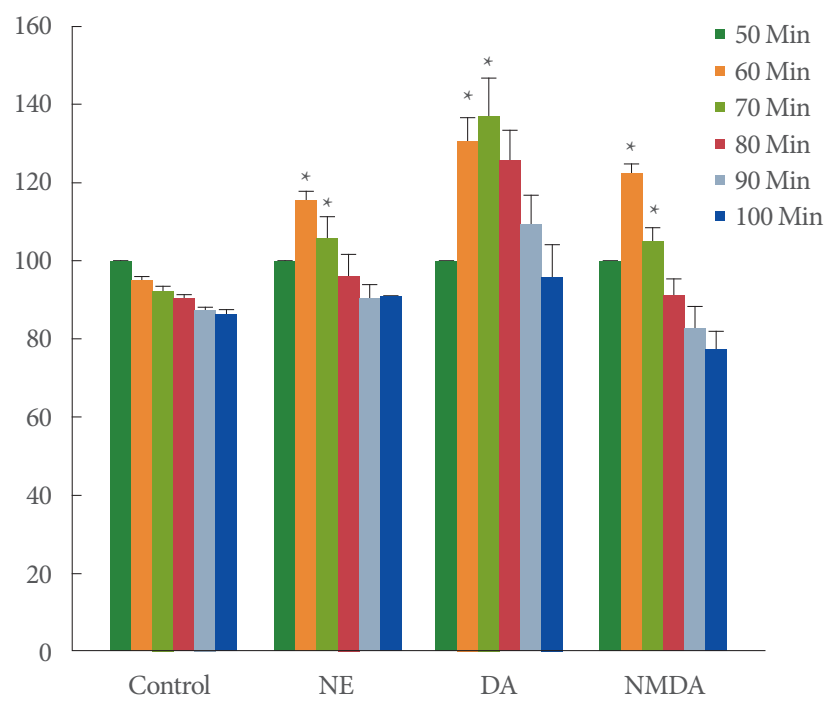

Fig. 2. The impact of norepinephrine (NE), dopamine (DA), and N-methyl-D-aspartate (NMDA) on 5-hydroxytryptamine (5-HT) discharge from rat hippocampal slices. The expression of every point takes the form of a percentage of the value at 50 minutes. Administration of the target pharmaceutical agents was done at the sixth and seventh 10-minute interval following the initial 50 minutes of the period of observation. 5-HT discharge was markedly enhanced by NE, DA, and NMDA by comparison to control of the sixth $(60 \mathrm{~min})$ and seventh 10-minute intervals $(70 \mathrm{~min})\left({ }^{*} \mathrm{P}<0.05\right)$. Expression of values takes the form of mean \pm standard error of the mean.
NMDA-induced rise in 5-HT discharge was inhibited when tetrodotoxin $\left(10^{-6} \mathrm{M}\right)$ treatment was applied before NMDA administration $(\mathrm{P}<0.05)$ (Figs. 2, 3).

\section{Ach-Induced Alteration in $\left.{ }^{3} \mathrm{H}\right] 5-\mathrm{HT}$ Discharge}

Ach treatment was applied to the incubation medium for 20 minutes to determine how 5-HT discharge from the slices of hippocampus was affected by the cholinergic neurotransmitter. The control group exhibited $\left[{ }^{3} \mathrm{H}\right] 5-\mathrm{HT}$ discharge of $94.9 \%$ $\pm 1.0 \%$ at the sixth 10 -minute interval and $92.3 \% \pm 1.1 \%$ at the seventh 10-minute interval. As shown in Fig. 4, the $\left[{ }^{3} \mathrm{H}\right] 5-\mathrm{HT}$ discharge from hippocampal slices was unaffected by Ach $\left(10^{-}\right.$ ${ }^{6} \mathrm{M}$ ) administration.

\section{$\left[{ }^{3} \mathrm{H}\right] 5-\mathrm{HT}$ Discharge Alteration Induced by GABa, Glycine, or Neuropeptide Y}

Analysis was also conducted on the influence of the amino acid neurotransmitters GABA and glycine and the peptide neurotransmitter neuropeptide $\mathrm{Y}$ on the unplanned discharge of 5-HT from rat hippocampal slices. Results indicated that those neurotransmitters had no effect on the discharge (Fig. 4).

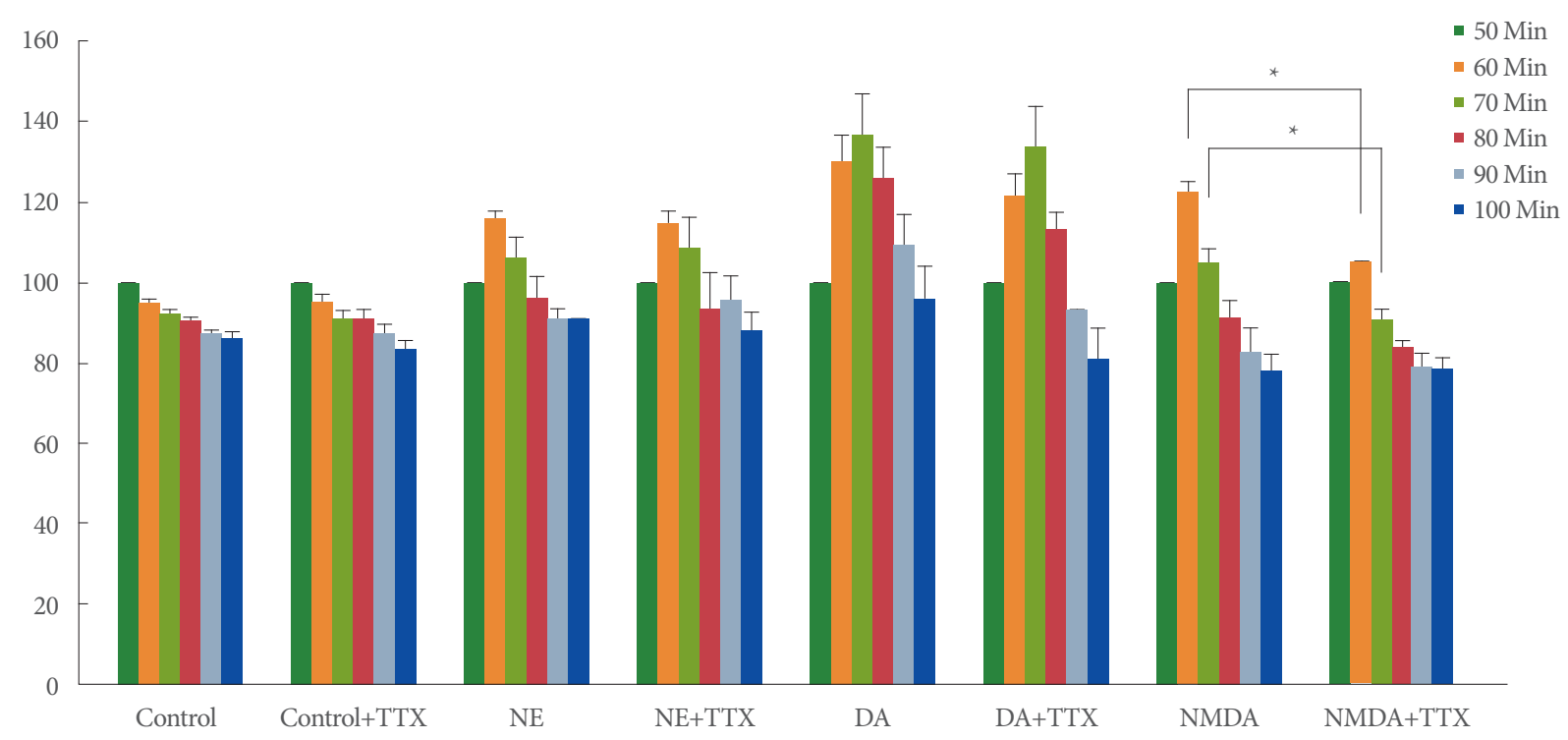

Fig. 3. Tetrodotoxin (TTX) impact on 5-hydroxytryptamine (5-HT) discharge from rat hippocampal slices triggered by norepinephrine (NE), dopamine (DA), and N-methyl-D-aspartate (NMDA). The expression of every point takes the form of a percentage of the value at 50 minutes. Administration of the target pharmaceutical agents was done at the sixth and seventh 10-minute interval following the initial 50 minutes of the period of observation, while administration of tetrodotoxin was continuous all through the empirical work. NMDA-induced 5-HT discharge was markedly suppressed by tetrodotoxin by comparison to control $\left({ }^{\star} \mathrm{P}<0.05\right)$. Expression of values takes the form of mean \pm standard error of the mean. 


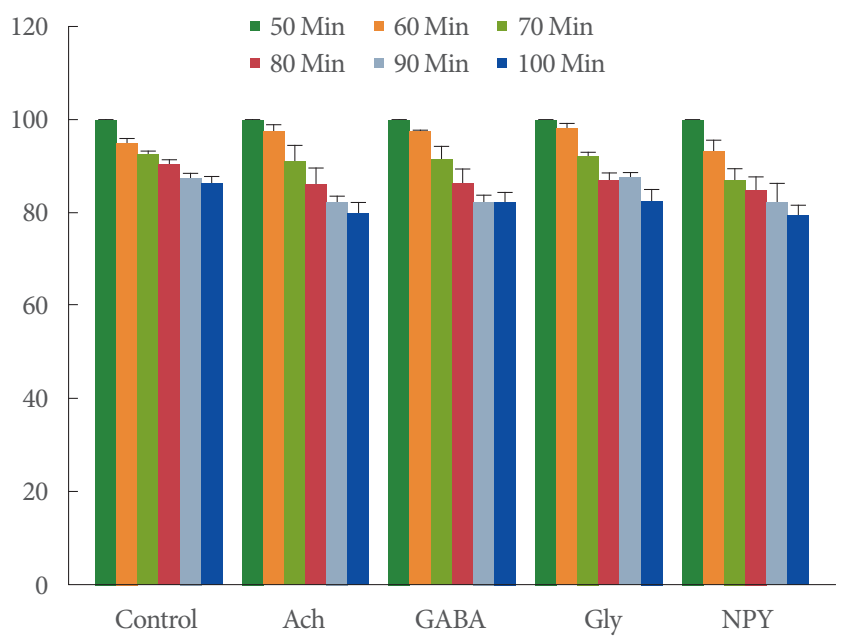

Fig. 4. The impact of acetylcholine, gamma-aminobutyric acid (GABA), glycine or neuropeptide Y on 5-hydroxytryptamine (5-HT) discharge from rat hippocampal slices. The expression of every point takes the form of a percentage of the value at 50 minutes. Administration of the target pharmaceutical agents was done at the sixth and seventh 10-minute interval following the initial 50 minutes of the period of observation. 5-HT discharge was not enhanced by acetylcholine, GABA, glycine, or neuropeptide $\mathrm{Y}$ comparison to control of the sixth (60 $\mathrm{min})$ and seventh 10-minute intervals (70 min). Expression of values takes the form of mean \pm standard error of the mean.

\section{DISCUSSION}

The upper medulla or pons is the main location where cathecholaminergic neurons are found. Micturition is regulated with the involvement of the spinal noradrenergic system, which blocks afferent input from the bladder and promotes enhanced bladder contractility by mediating the descending limb of the spinal micturition reflex $[14,15]$. Micturition is suppressed by dopamine via D1-like receptors and stimulated via D2-like receptors [16]. Entering from the locus coeruleus, noradrenalinergic nerve fibers alongside serotonergic nerve fibers traverse most of the hippocampus, particularly the dentate gyrus. Furthermore, the hippocampus and subiculum contain dopaminergic nerve fibers from the ventral segmental region. The present work discovered that norepinephrine and dopamine differed markedly regarding 5-HT discharge [15,16].

By comparison to control, both the norepinephrine $\left(10^{-5} \mathrm{M}\right)$ group and the dopamine $\left(10^{-5} \mathrm{M}\right)$ group differed markedly in terms of 5-HT discharge. This supported the conclusion that 5-HT discharge could be influenced by norepinephrine or dopamine. Furthermore, by comparison to norepinephrine, dopa- mine induced the discharge of more 5-HT, therefore having a greater influence (Fig. 2). What is more, prior administration of tetrodotoxin did not inhibit the enhanced 5-HT discharge from the slices of hippocampus triggered by either norepinephrine or dopamine. Therefore, it was deduced that the serotoninergic nerve fiber terminals were impacted by the noradrenalinergic and dopaminergic nerve fibers in a direct manner, without interneuron mediation (Fig. 3).

It is considered that glutamate is the main neurotransmitter in Barrington nucleus neurons innervation the preganglionic parasympathetic neurons that cause the detrusors to contract $[1,3]$. Furthermore, it seems that glutamate serves as excitatory transmitter in the supraspinal pathway that regulates micturition. Alongside interneurons and fibers stemming from the medulla oblongata, glutamate occurs within the terminals of primary afferent neurons in the spinal cord. NMDA receptors are among the different subtypes of receptors through which glutamate exerts its effect on spinal neurons $[1,3]$. As a standard excitatory amino acid neurotransmitter, glutamate influences 5-HT discharge from the terminal of serotonergic nerve fibers [17].

Exerting its effect via the NMDA receptor, the excitatory amino acid neurotransmitter glutamate was observed in this work to impact 5-HT discharge from the terminal of serotonergic nerve fibers (Fig. 2). By comparison to control, the group administered NMDA $\left(10^{-4} \mathrm{M}\right)$ exhibited higher 5-HT discharge from rat hippocampal slices, leading to the conclusion that 5-HT discharge regulation may depend on NMDA. Meanwhile, as shown in Fig. 3, the rise in 5-HT discharge from hippocampal slices was inhibited in the context of prior administration of tetrodotoxin. Therefore, 5-HT discharge from rat hippocampal slices is influenced by the excitatory amino acid neurotransmitter NMDA, which acts via interneuron mediation rather than straight on the glutamatergic nerve terminals.

The micturition reflex in the spinal cord is suppressed by muscarinic Ach receptors. Meanwhile, it has been observed that the rat micturition reflex is stimulated by nicotine administered intrathecally, meaning that the voiding function is regulated with the participation of nicotinic receptors [18]. As illustrated in Fig. 4, the present work revealed that the Ach $\left(10^{-5} \mathrm{M}\right)$ group and the control group did not differ markedly in terms of 5-HT discharge, implying that the serotonergic nerve fiber is not influenced by the cholinergic nerve fiber.

The dentate gyrus, hippocampus, and subiculum make up the hippocampal formation. Immunoreactive cells with sensi- 


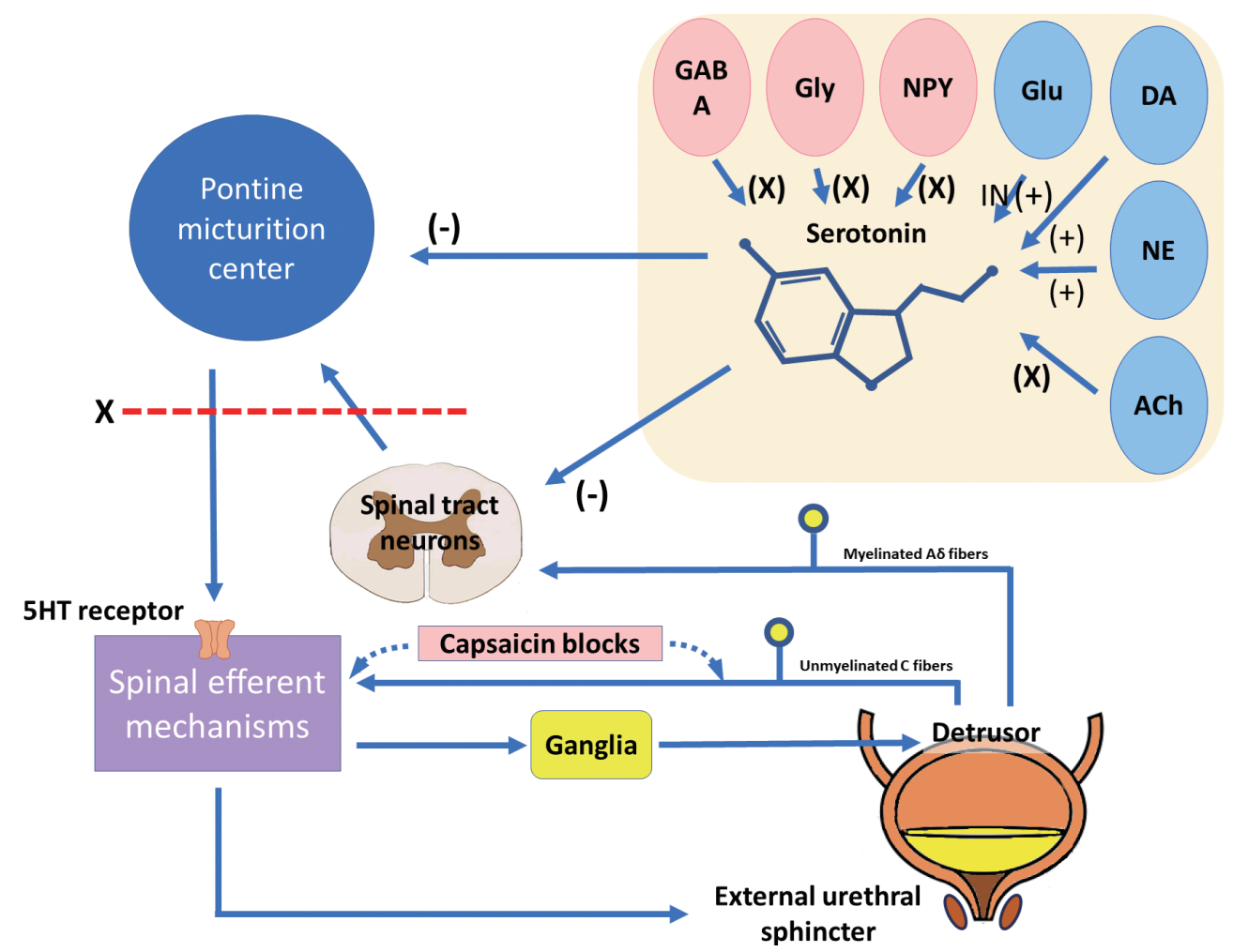

Fig. 5. The involvement of different additional neurotransmitters in 5-hydroxytryptamine (5-HT) discharge from the hippocampal slices of rats. 5-HT discharge is enhanced by norepinephrine and dopamine through direct action on the 5-HT neuronal terminal. Meanwhile, 5-HT discharge is indirectly enhanced by NMDA via interneuron mediation. GABA, gamma-aminobutyric acid; NPY, neurotransmitter neuropeptide Y; Gly, glycine, Glu, glutamate; DA, dopamine; NE, norepinephrine; Ach, acetylcholine; NMDA, Nmethyl-D-aspartate; $\mathrm{X}$, no effect on the discharge.

tivity to somatostatin and GABA are found in the polymorphic layers of the dentate gyrus and give rise to associational fibers [19]. Meanwhile, interneurons derive from the numerous nonpyramidal cells in the hippocampal CA3 region. However, understanding of how they are connected has not been achieved so far [20]. The interneurons of the dentate gyrus, CA1, subiculum, and entorhinal cortex all contain neuropeptide Y [10].

Neuronal regulatory mechanisms underpinning bladder function depend significantly on glycinergic and GABAergic interneurons. Both the micturition reflex and glutamatergic neurons are suppressed by glycinergic and GABAergic branches to the lumbosacral cord [21]. The present work observed that, by comparison to control, 5-HT discharge from hippocampal slices was more or less the same in the groups administered the inhibitory amino acid neurotransmitters GABA $\left(10^{-5} \mathrm{M}\right)$ or glycine $\left(10^{-5} \mathrm{M}\right)$ found within hippocampal formation interneurons (Fig. 4). Similar findings were obtained regarding the peptide neurotransmitter neuropeptide $\mathrm{Y}\left(10^{-5} \mathrm{M}\right)$ as well (Fig. 4). This led to the conclusion that 5-HT discharge was not regulat- ed by GABA, glycine, or neuropeptide Y via interneuron mediation.

By contrast to Ach, GABA, glycine, and neuropeptide Y, the results indicated that norepinephrine, DOPA, and NMDA influenced 5-HT discharge from the hippocampus (Fig. 5).

In conclusion, the current work defended the premise that a range of different neurons was involved in 5-HT discharge from rat hippocampal slices. More specifically, 5-HT discharge was intensified by norepinephrine and dopamine through direct action on the 5-HT neuronal terminal. Meanwhile, 5-HT discharge was intensified by NMDA in an indirect manner, via interneuron mediation.

\section{AUTHOR CONTRIBUTION STATEMENT}

- Conceptualization: YSS

- Data curation: JHK, YSA

- Formal analysis: YSA, YSS

- Funding acquisition: YSS 
- Methodology: JHK, YSA

- Project administration: YSS

- Visualization: YSA

-Writing-original draft: JHK, YSS

-Writing-review \& editing: YSA, YSS

\section{ORCID}

Jae Heon Kim

0000-0002-4490-3610

Yun Seob Song

0000-0002-0909-3341

\section{REFERENCES}

1. Chang HY, Cheng CL, Chen JJ, de Groat WC. Roles of glutamatergic and serotonergic mechanisms in reflex control of the external urethral sphincter in urethane-anesthetized female rats. Am J Physiol Regul Integr Comp Physiol 2006;291:R224-34.

2. de Groat WC, Yoshimura N. Pharmacology of the lower urinary tract. Annu Rev Pharmacol Toxicol 2001;41:691-721.

3. Yoshiyama M, De Groat WJN. Supraspinal and spinal a-amino-3hydroxy-5-methylisoxazole-4-propionic acid and N-methyl-d-aspartate glutamatergic control of the micturition reflex in the urethane-anesthetized rat. Neuroscience 2005;132:1017-26.

4. Chen SY, Wang SD, Cheng CL, Kuo JS, De Groat WC, Chai CY. Glutamate activation of neurons in CV-reactive areas of cat brain stem affects urinary bladder motility. Am J Physiol 1993;265(4 Pt 2):F520-9.

5. de Groat WC, Griffiths D, Yoshimura N. Neural control of the lower urinary tract. Compr Physiol 2015;5:327-96.

6. McMahon SB, Spillane K. Brain stem influences on the parasympathetic supply to the urinary bladder of the cat. Brain Res 1982; 234:237-49.

7. Garraway SM, Hochman S. Modulatory actions of serotonin, norepinephrine, dopamine, and acetylcholine in spinal cord deep dorsal horn neurons. J Neurophysiol 2001;86:2183-94.

8. Blok BF, Holstege G. The central nervous system control of micturition in cats and humans. Behav Brain Res 1998;92:119-25.

9. Tadic SD, Tannenbaum C, Resnick NM, Griffiths D. Brain responses to bladder filling in older women without urgency inconti- nence. Neurourol Urodyn 2013;32:435-40.

10. Chan-Palay V, Kohler C, Haesler U, Lang W, Yasargil G. Distribution of neurons and axons immunoreactive with antisera against neuropeptide $\mathrm{Y}$ in the normal human hippocampus. J Comp Neurol 1986;248:360-75.

11. Moore RY, Halaris AE. Hippocampal innervation by serotonin neurons of the midbrain raphe in the rat. J Comp Neurol 1975;164: 171-83.

12. Becquet D, Faudon M, Hery F. In vivo evidence for an inhibitory glutamatergic control of serotonin release in the cat caudate nucleus: involvement of GABA neurons. Brain Res 1990;519:82-8.

13. Schiff SJ, Somjen GG. The effects of temperature on synaptic transmission in hippocampal tissue slices. Brain Res 1985;345:279-84.

14. Yoshimura N, Sasa M, Ohno Y, Yoshida O, Takaori S. Contraction of urinary bladder by central norepinephrine originating in the locus coeruleus. J Urol 1988;139:423-7.

15. Yoshimura N, Sasa M, Yoshida O, Takaori S. Mediation of micturition reflex by central norepinephrine from the locus coeruleus in the cat. J Urol 1990;143:840-3.

16. Hashimoto K, Oyama T, Sugiyama T, Park YC, Kurita T. Neuronal excitation in the ventral tegmental area modulates the micturition reflex mediated via the dopamine D1 and D2 receptors in rats. J Pharmacol Sci 2003;92:143-8.

17. Matsumoto G, Hisamitsu T, de Groat WC. Role of glutamate and NMDA receptors in the descending limb of the spinobulbospinal micturition reflex pathway of the rat. Neurosci Lett 1995;183:5861.

18. Masuda H, Hayashi Y, Chancellor MB, Kihara K, de Groat WC, de Miguel F, et al. Roles of peripheral and central nicotinic receptors in the micturition reflex in rats. J Urol 2006;176:374-9.

19. Ribak CE, Seress L. Five types of basket cell in the hippocampal dentate gyrus: a combined Golgi and electron microscopic study. J Neurocytol 1983;12:577-97.

20. Amaral DG, Campbell MJ. Transmitter systems in the primate dentate gyrus. Hum Neurobiol 1986;5:169-80.

21. Matsuta Y, Yusup A, Tanase K, Ishida H, Akino H, Yokoyama O. Melatonin increases bladder capacity via GABAergic system and decreases urine volume in rats. J Urol 2010;184:386-91. 